\title{
Chloramphenicol-resistant Salmonella tennessee Osteomyelitis
}

\section{Chloramphenicol-resistant Salmonella tennessee Osteo- myelitis}

Localized infection following Salmonella septicemia is most often seen in immunocompromised patients (1). In the immunosuppressed patient, treatment of Salmonellarelated infectious lesions is vital and may cause major problems (2). It is of clinical importance to carefully monitor treatment-resistant and rare Salmonella species in these patients.

In the FRG, only $0.05 \%$ of Salmonella species isolated from patients' blood or tissues are found to be chloramphenicol-resistant (3). If such resistance occurs and neither ampicillin nor co-trimoxazole can provide therapeutic concentrations at the site of local infection, there is great need for a drug with both a well defined tissue permeability and a clinically proven therapeutic effect. To illustrate this therapeutic dilemma, we would like to report a case of osteomyelitis following septicemia. Salmonella tennessee, the causative agent in this infection, was found to be chloramphenicol-resistant.

\section{Case Report}

A $4 \frac{1}{2}$ year-old girl with neuroblastoma stage IV disease presented with a two-week history of arthralgia (left hip, right shoulder) and febrile episodes (up to $41^{\circ} \mathrm{C}$ ). Several blood cultures grew $S$. tennessee as the single agent. The child was started on chloramphenicol $(100 \mathrm{mg} / \mathrm{kg} /$ day $)$. However, she remained febrile and a Tc-99m-DPD-bone scintigraphy was carried out. Multiple focal lesions (left femur, vertebral column, left cranium) were detected. Subsequently, specific ${ }^{131}$ I-MIBG $\left({ }^{131}\right.$ I-meta-iodo-benzylguanidine) scintigraphy was performed. Specific accumulation of ${ }^{131}$ I-MIBG was found in the left side of the cranium and in the right paravertebral region. Subsequent Xray revealed a large osteomyelitic lesion in the left distal femur which had to be treated surgically: a large sequester was removed and $S$. tennessee was cultivated from the necrotic material. Histological examination of the sequester did not reveal neuroblastoma cells. Thus the scintigraphic findings in the cranium were interpreted as neuroblastoma bone lesions, whereas infectious osteomyelitis was suspected in the left femur and vertebral column.

\section{Microbiology}

The agar diffusion technique revealed an ampicillin and chloramphenicol-sensitive Salmonella species. However, clinically the child did not respond to high-dose chloramphenicol (up to $150 \mathrm{mg} / \mathrm{kg} / \mathrm{day}$ ), and the MICs of chloramphenicol were found to range from 2.5 to $8.0 \mathrm{mg} / 1$ (our own laboratory results were confirmed by tests at the Institute of Hygiene, Department of Medical Microbiology,
University of Frankfurt/M, FRG, Prof. Dr. H. Knothe). (The MIC of ampicillin was $2.5 \mathrm{mg} / \mathrm{ml}$. The MIC of co-trimoxazole was not tested). S. tennessee, the causative agent, was isolated from two subsequent blood cultures and from necrotic tissue (one needle aspirate from the left distal femur and from the surgically removed sequestra). Despite vigorous efforts, the origin of the infection could not be traced. However, it is of interest that the girl had lived at her parents' farm until she became ill.

After formal parental consent and approval by the hospital's ethic commission, the child was consequently started on ciprofloxacin, a new quinoline carboxylic acid derivative $(100 \mathrm{mg} /$ day). Under this experimental treatment the child became afebrile, the bone lesion improved and the child could be discharged home on oral cyclophosphamide after nine weeks.

\section{Discussion}

The case of a child with stage IV neuroblastoma and additional septicemia due to chloramphenicol-resistant $S$. tennessee is reported. It is of clinical interest that this case of otherwise rare Salmonella infection $(0.05 \%$ of all Salmonella cases in the FRG [3]) occurred in a patient whose bone marrow function was disturbed as a consequence of malignancy (diffuse infiltration of bone marrow by neuroblastoma cells). This finding is consistent with unusual Salmonella infections reported in a large series of patients with neoplastic disease in the U.S.A. (1).

In our case, commercially available drugs could not solve the therapeutic dilemma, and experimental treatment achieved an excellent clinical result. However, so far long-term effects of ciprofloxacin in humans are not known. Until further investigations, chloramphenicol-resistant Salmonella species will cause rare but serious therapeutic problems.

W. Kiess, R. Haas, W. Marget

\section{Literature}

1. Wolfe, M. S., Armstrong, D., Louria, D. B., Blevins, A.: Salmonellosis in patients with neoplastic disease. Arch. Intern. Med. 128 (1971) 546-554.

2. Anderson, R. J.: Infectious risk factors in the immunosuppressed host. Am. J. Med. 54 (1973) 453-460.

3. Knothe, H.: Personal communication (1984).

W. Kiess, R. Haas, W. Marget, Children's Hospital, University of Munich, Lindwurmstr. 4, D-8000 München 2. 